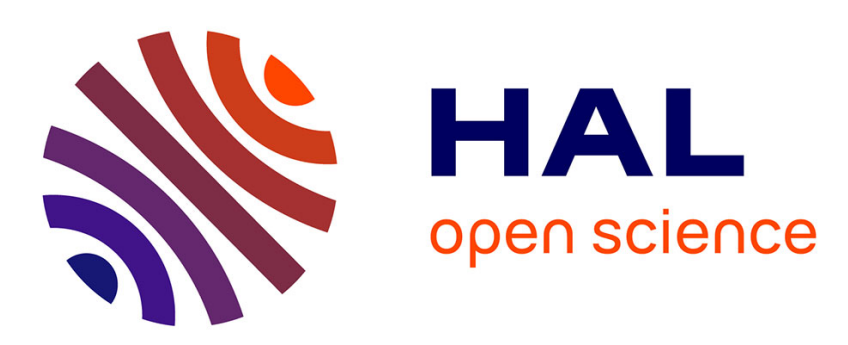

\title{
Order parameter and detection for a finite ensemble of crystallized one-dimensional dipolar bosons in optical lattices
}

Budhaditya Chatterjee, Axel Lode

\section{- To cite this version:}

Budhaditya Chatterjee, Axel Lode. Order parameter and detection for a finite ensemble of crystallized one-dimensional dipolar bosons in optical lattices. Physical Review A, 2018, 98, pp.053624. 10.1103/PhysRevA.98.053624 . hal-02370257

\section{HAL Id: hal-02370257 https://hal.science/hal-02370257}

Submitted on 19 Nov 2019

HAL is a multi-disciplinary open access archive for the deposit and dissemination of scientific research documents, whether they are published or not. The documents may come from teaching and research institutions in France or abroad, or from public or private research centers.
L'archive ouverte pluridisciplinaire HAL, est destinée au dépôt et à la diffusion de documents scientifiques de niveau recherche, publiés ou non, émanant des établissements d'enseignement et de recherche français ou étrangers, des laboratoires publics ou privés. 


\title{
Order parameter and detection for a finite ensemble of crystallized one-dimensional dipolar bosons in optical lattices
}

\author{
Budhaditya Chatterjee ${ }^{1, *}$ and Axel U. J. Lode Lo $^{2,3,4, \dagger}$ \\ ${ }^{1}$ Department of Physics, Indian Institute of Technology-Kanpur, Kanpur 208016, India \\ ${ }^{2}$ Wolfgang Pauli Institute c/o Faculty of Mathematics, University of Vienna, Oskar-Morgenstern Platz 1, 1090 Vienna, Austria \\ ${ }^{3}$ Vienna Center for Quantum Science and Technology, Atominstitut, TU Wien, Stadionallee 2, 1020 Vienna, Austria \\ ${ }^{4}$ Department of Physics, University of Basel, Klingelbergstrasse 82, CH-4056 Basel, Switzerland
}

(Received 9 September 2017; published 26 November 2018)

\begin{abstract}
We explore the ground-state properties of a few bosons with dipole-dipole interactions in a one-dimensional optical lattice. For comparatively strong interactions, a transition from a Mott-insulator state to a crystal state occurs. Herein, we provide a detailed characterization and a detection protocol for the resulting crystal state. Using the eigenvalues of the reduced one-body density matrix, we define an order parameter that characterizes all the emergent states occurring in the finite-sized ensemble and agrees with the signatures obtained from the analysis of the one- and two-body densities. We further demonstrate that these few-body emergent states can be detected experimentally using the variance of single-shot measurements.
\end{abstract}

DOI: 10.1103/PhysRevA.98.053624

\section{INTRODUCTION}

Dipolar ultracold atoms have attracted much interest recently $[1,2]$. This interest is corroborated by experimental realizations of dipolar Bose-Einstein condensates (BECs) of chromium [3,4], dysprosium [5], and erbium [6] atoms as well as potassium-rubidium molecules [7]. The realization of ultracold sodium-potassium molecules with tunable dipolar interaction strength enables the exploration of a regime where dipolar interactions are dominant [8].

The long-range and anisotropic dipole-dipole interaction leads to rich and exotic quantum effects distinct from the effects of BECs with contact interactions. Dipolar bosons in a trap show an elongation of the condensate along the direction of the orientation of the dipoles [9-11]. Counterintuitively, the stability of dipolar condensates increases in special geometries like very oblate traps $[9,10,12-14]$. The long-range and anisotropic interactions make dipolar ultracold atoms a great resource to explore quantum phases and aspects of many-body physics [2,15-17].

In contrast to condensed matter setups, the parameters of cold-atom systems can be controlled almost at will. Changing the dimensionality may yield additional features such as $p$-wave superfluidity in two-dimensional Fermi gases [18,19], Luttinger-liquid-like behavior in onedimensional bosons [20-23] and an anisotropy effect for bosons on a ring [24-26].

Even few-particle systems can be deterministically produced in experiments [27] and enable the investigation of the fundamental building blocks of many-body systems, for instance, in lattices $[28,29]$ from a bottom-up perspective. Moreover, these few-atom systems can be handled numer-

\footnotetext{
*bchat@iitk.ac.in

†axel.lode@univie.ac.at
}

ically accurately with any precision for any interparticle interaction strength $[30,31]$ allowing the investigations of properties emergent for strong interactions, like, for instance, fermionization in lattices [32-34].

Ultracold atoms in lattices can serve as a quantum simulator for condensed matter systems [35-38]. Dipolar atoms in optical lattices, due to their long-range anisotropic interactions, have an enriched phase diagram as compared to systems with contact interactions: a density-wave phase $[15,39]$ and Haldane insulating phases $[39,40]$ were predicted in one- and two-dimensional systems.

In this work, we focus on a remarkable property that renders strongly interacting dipolar systems significantly different from atoms with contact interactions: a crystallization process is seen for one-dimensional homogeneous dipoles [20], dipoles in a linear or a zigzag chain $[41,42]$, in a harmonic trap [43], a ring geometry [24,25], and in a triple well [44]. A similar crystallization phenomenon has also been seen for other strongly correlated nondipolar systems [45].

Theoretically, dipolar atoms in triple wells have, for instance, been investigated using mean-field methods [46], the Hubbard model [47-51], and using the multiconfigurational time-dependent Hartree (MCTDH) method [44]. The physics of strong dipolar interactions lies beyond the area of validity of mean-field methods and standard Hubbard models [24,25,44,52-55]; the usage of a general many-body approach is necessary. We follow the strategy of Refs. [27-29] and determine the physics of the many-body system from the analysis and understanding of its few-body building blocks. We solve the few-body Schrödinger equation for the ground states of bosons with dipole-dipole interactions in a multiwell potential using the MCTDH for bosons (MCTDHB) method [56] implemented in the MCTDH-X software $[31,57,58]$. Chiefly, we investigate a triple well because it is the elemental building block that exhibits all essential long-range dipolar effects of bosons in optical lattices. 
We theoretically explore as a function of an increasing dipole-dipole interaction strength the few-body ground states which are the finite-sized precursors to the thermodynamic phases. We analyze the one- and two-body densities and identify the characteristic density signatures for all the emergent phases, the superfluid (SF), the Mott insulator (MI), and the crystal state (CS). We simulate the emergence of these phases as a function of the strength of the dipolar interaction and the depth of the lattice potential for commensurate fillings, thereby effectively computing an analogous few-body phase diagram. We demonstrate how the natural populations, i.e., the eigenvalues of the reduced one-body density matrix, can be used to define a mesoscopic "order parameter" that unequivocally characterizes these states and thus yields a few-body equivalent of a "phase diagram." We further show that these few-body phases can be straightforwardly measured using the variance of images obtained from single-shot measurements.

The paper is structured as follows: In Sec. II, we discuss Hamiltonian and the setup of our system. In Sec. III, we explore and analyze the one- and two-body density signatures of the emergent phases. In Sec. IV, we discuss the construction of the order parameter and compute the phase diagram. In Sec. V, we explain and discuss the experimental detection protocol using single-shot measurements. Section VI concludes the paper.

\section{SETUP}

We consider $N$ dipolar bosons in a quasi-one-dimensional optical lattice with all their dipoles polarized along the same direction:

$$
H=\sum_{i=1}^{N}-\frac{\hbar^{2}}{2 M} \partial_{x_{i}}^{2}+\sum_{i=1}^{N} V_{o l}\left(x_{i}\right)+\sum_{i<j} V_{\mathrm{int}}\left(x_{i}-x_{j}\right) .
$$

The optical lattice $V_{o l}=V \sin ^{2}(\kappa x)$ has the depth $V$ and the wave vector $\kappa$. We consider $S$ lattice sites with hardwall boundary conditions. A strong transverse confinement of characteristic length $a_{\perp}$ prevents any excitation into the transverse direction and ensures the quasi-one-dimensionality.

In the absence of $s$-wave scattering, the quasi-onedimensional dipole-dipole interaction is modeled as $V_{\mathrm{int}}\left(x_{i}-\right.$ $\left.x_{j}\right)=g_{c} \delta\left(x_{i}-x_{j}\right)+\frac{g_{d}}{\left|x_{i}-x_{j}\right|^{3}+\alpha}\left(g_{c}, g_{d}\right.$ are the coupling constants). For large separations $r=\left|x_{i}-x_{j}\right| \gg a_{\perp}$, we get the far-field long-range dipole-dipole interaction $\sim 1 / r^{3}$. For small separations $\left|x_{i}-x_{j}\right| \lesssim a_{\perp}$, the transverse confinement regularizes the divergence at $x_{i}=x_{j}$ by introducing an effective interaction cutoff $\alpha \approx a_{\perp}{ }^{3}$ and an additional $\delta$-like interaction $g_{c} \delta\left(x_{i}-x_{j}\right)[43,59,60]$. The coupling constants are related as $g_{c} \approx g_{d} \frac{4 \sqrt{\gamma}}{3 \sqrt{2 \pi}}$, where $\gamma$ is the trap aspect ratio $\frac{a_{\|}}{a_{\perp}}$ [60]. We henceforth refer to $g_{d}$ as the strength of the interaction and is given as $g_{d}=d_{m}^{2} / 4 \pi \epsilon_{0}$ for electric dipoles and as $g_{d}=d_{m}^{2} \mu_{0} / 4 \pi$ for magnetic dipoles, where $d_{m}$ is the dipole moment, $\epsilon_{0}$ the vacuum permittivity, and $\mu_{0}$ the vacuum permeability.

To arrive at convenient dimensionless units, we rescale the Hamiltonian in Eq. (1) by the lattice recoil energy $E_{R}=\hbar^{2} \kappa^{2} / 2 M$ and investigate a triple-well setup $(S=3)$ with hard-wall boundaries at $x= \pm S \pi / 2 \kappa$, an aspect ratio $\gamma=25.6$, and an interaction cutoff $\alpha=0.05$. Results for other parameters are shown in Appendix A. Since commensurability is necessary to realize the aforementioned phases, we will discuss exclusively commensurate fillings.

\section{DENSITY SIGNATURES OF THE EMERGENT PHASES}

For bosons with contact interactions in a lattice, the competition between the kinetic and interaction energy determines the quantum phases [61,62]. For dipolar atoms, however, this competition is changed [54,63]: the relative strengths of the short-ranged portion and the long-ranged portion of the interaction affect the ground-state properties in addition to the kinetic energy. This is particularly important at large dipolar interaction strengths $g_{d}$ [64].

Let us clarify our adoption of the terminology "phases" and "order parameter." Rigorously, the concept of quantum phases and, likewise, their order parameter is legitimate solely in the thermodynamic limit. The finite-size ensemble we consider cannot exhibit true macroscopic phases in this strict sense. Nonetheless, for small ensembles, the ground-state possesses properties that are analogous to the macroscopic phases. These analogous properties are the "finite-size precursors" to the macroscopic quantum phase [65]. In our work, finite-size effects are still present but do not dictate the physics; for the sake of simplicity, we thus use the terms "phase" and "order parameter" when discussing the finite-size precursors of the quantum phase in the following.

We first analyze the one-body density $\rho(x)=$ $\left\langle\Psi\left|\hat{\Psi}^{\dagger}(x) \hat{\Psi}(x)\right| \Psi\right\rangle$ of the ground state as a function of the repulsive dipolar interaction strength $g_{d}$ for a fixed depth $V$ of the lattice [Fig. 1(a)]. For small interactions $g_{d} \approx 0$, the kinetic energy dominates the competition of energies and the bosons are in a coherent superposition of all number states (i.e., states counting the occupation of each lattice site). This fully delocalized state represents the superfluid phase. Dominating kinetic energy and hard-wall boundary conditions lead to a maximal population in the central well [Fig. 1(b)].

As the interaction strength increases, the short-range portion of the interaction begins to dominate, and the bosons localize in each well. The density exhibits a single maximum in each well. This Mott-insulator phase is characterized by the localization of atoms in the lattice with a vanishing overlap of the densities in distinct wells [Fig. 1(c)].

When the interaction strength increases further, the shortrange interactions become strong enough for the bosons to attempt to fermionize; a characteristic dip in the center of the one-body density in each well emerges [Fig. 1(d)]. Fermionization refers to systems resembling a Tonks gas for which the density is identical to the density of noninteracting fermions [66]. The original Bose-Fermi map is valid only for contact interactions. For dipolar interactions, a similar mapping can be constructed by exploiting the divergence of the interaction potential when the positions of two atoms become equal $[42,67]$.

In the strongly interacting limit, the long-ranged $\sim 1 / r^{3}$ tail of the dipolar interaction becomes dominant and determines the physics. As a consequence of the long-range interactions, well-separated density maxima for each particle in the system emerge [Fig. 1(e)]; this localized structure of the density is 

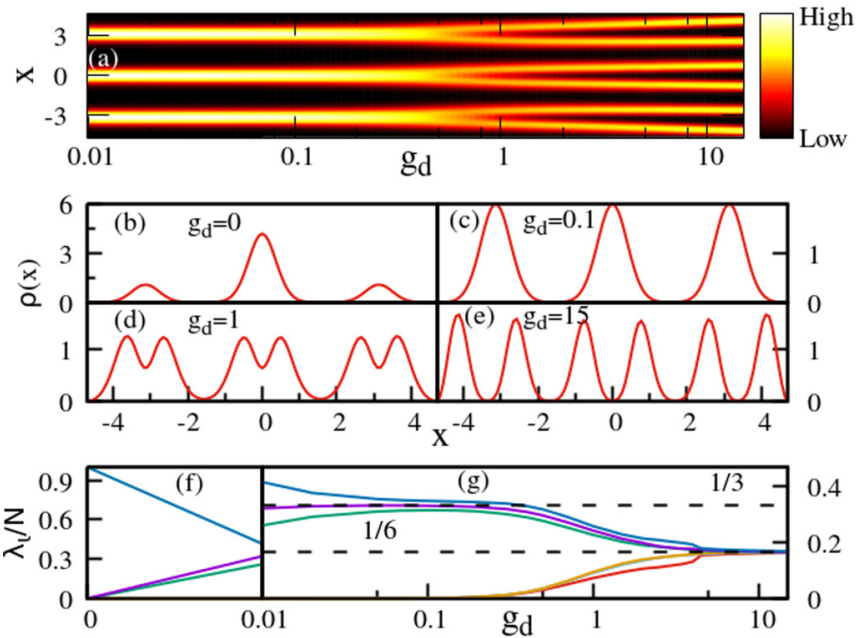

FIG. 1. (a) The one-body density $\rho(x)$ for $N=6$ bosons as a function of the strength of the dipolar interactions $g_{d}$. The depth of the lattice is fixed at $V=8$. The density shows a transition from a threefold to a sixfold spatial splitting as the dipolar interaction strength increases. (b)-(e) One-body densities representative for different phases. For the superfluid phase (b) the kinetic and trap energies dominate: the density is maximal in the central well. For the Mott-insulator phase (c) the bosons are equally distributed between all wells. In (d) the onset of fermionization and formation of the Tonks gas is mimicked by the formation of a characteristic dip at the center of each well and in (e) the emergence of wellseparated density peaks is one of the hallmarks of the crystal phase. (f), (g) Normalized population of natural orbitals as a function of the interaction strength $g_{d}$. At $g_{d} \approx 0$, only the first orbital has a significant population. As the interaction increases, $n=3$ orbitals begin to populate reaching an equal population in the Mott-insulator state. For large $g_{d}$ as many orbitals as there are particles $(N=6)$ are populated, and the system reaches the maximally fragmented crystal state.

a feature of the crystal phase and marks the departure of the physics of the system from the area of validity of the Hubbard model.

Next, we illustrate the mechanism of the localization process for the different phases using the two-body density $\hat{\rho}_{2}\left(x_{1}, x_{2}\right)=\left\langle\Psi\left|\hat{\Psi}^{\dagger}\left(x_{1}\right) \hat{\Psi}^{\dagger}\left(x_{2}\right) \hat{\Psi}\left(x_{1}\right) \hat{\Psi}\left(x_{2}\right)\right| \Psi\right\rangle$ (Fig. 2).

As interactions increase, the transition from the superfluid to the Mott state (Fig. 2, upper row) is seen from the change in $\rho_{2}$ from a maximum at center $x_{1}=x_{2}=0$ to off-diagonal $x_{1} \neq x_{2}$ maxima and a depletion of its diagonal: the atoms start to minimize the probability to be at the same position in space while remaining in the lowest band of the lattice [Fig. 2 $\left.\left(g_{d}=0.1\right)\right]$. With a further increase of the interaction strength, $\rho_{2}$ develops a correlation hole, i.e., $\rho_{2}(x, x) \rightarrow 0$, implying that the probability of finding two bosons in the same place is reduced [Fig. $\left.2\left(g_{d}=1.0\right)\right]$. This resembles the behavior of hard-core bosons with infinitely strong contact interactions [cf. Fig. 1(d)].

Eventually, when the dipolar interaction strength is increased beyond the Tonks regime [Fig. $\left.2\left(g_{d}=15\right)\right]$, the longrange tail of the interaction determines the physics of the system: the two-body density shows a complete spatial isolation of every particle in a square-lattice-like pattern with a missing

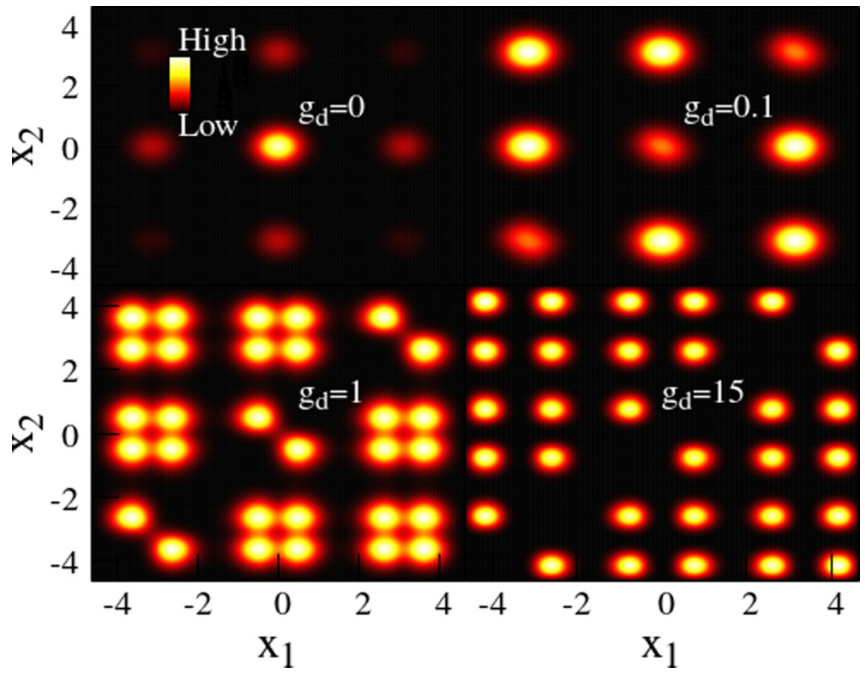

FIG. 2. Exploring the two-body density in the emergent phases. In the absence of dipole-dipole interactions $\left(g_{d}=0\right)$, the atoms cluster at $\left(x_{1}=x_{2}=0\right)$. The delocalization of the atoms in the superfluid causes the noncentral peaks to locate at $x_{1}=0, x_{2}= \pm \pi$ and $x_{1}= \pm \pi, x_{2}=0$ while the central peak is at $x_{1}=x_{2}=0$. At $g_{d}=0.1$, the localization of atoms in the Mott-insulating phase is evident from the depletion of the diagonal of the two-body density. At $g_{d}=1.0$, as the dipoles fermionize as a "correlation hole" develops: the two-body density tends to zero for $x_{1}=x_{2}$. At $g_{d}=15$, the dipoles crystallize and the two-body density forms a square lattice with missing diagonal.

diagonal; a hallmark of the transition from the fermionized gas to the crystal state. The crystal state is a pure longrange interaction effect and cannot be reached with contact interactions alone [cf. Fig. $2\left(g_{d}=15\right)$, Fig. 7 in Ref. [32]].

\section{ORDER PARAMETER AND PHASE DIAGRAM}

We now show that the eigenvalues of the reduced one-body density matrix or natural populations can be used to define an effective few-body order parameter that identifies all the aforementioned phases of few dipolar atoms in finite optical lattices.

The reduced one-body density matrix is defined as

$$
\hat{\rho}_{1}\left(x, x^{\prime}\right)=\left\langle\Psi\left|\hat{\Psi}^{\dagger}(x) \hat{\Psi}\left(x^{\prime}\right)\right| \Psi\right\rangle=\sum_{i} \lambda_{i} \varphi_{i}^{*}(x) \varphi_{i}\left(x^{\prime}\right) .
$$

The second equality illustrates that $\rho_{1}$ can be diagonalized to yield an expansion in terms of its eigenfunctions $\varphi_{i}(x)$, and its eigenvalues $\lambda_{i}$, also termed natural orbitals and occupations, respectively.

If only one natural population $\lambda_{i}$ is macroscopic, the system is condensed [68], and if several natural populations $\lambda_{i}$ are macroscopic, the system is said to be fragmented $[69,70]$.

For small interactions, the bosons are condensed and form a superfluid; only the lowest natural orbital is populated and only the first natural population is macroscopic, $\lambda_{1} \approx N$ [Fig. 1(f)].

With the transition to the Mott-insulating phase, fragmentation emerges: the reduced one-body density matrix attains as many equally large eigenvalues as there are lattice sites, while 
all other eigenvalues are zero [Fig. 1(g)]. In a system of $N$ particles in $S$ sites, the significant eigenvalues of the reduced density matrix are hence equal to $N / S: \lambda_{i} \approx N / S$ for $i \leqslant S$ and $\lambda_{i} \approx 0$ for $i>S$.

As interactions increase beyond the Mott-insulating phase, more natural orbitals become populated until fragmentation is maximal and the crystal state forms: $N$ orbitals attain unit population, irrespective of the number of sites $S$ [Fig. 1(g)].

In order to use our above observations on the one-body and two-body densities and the natural occupations to study the phase diagram of ultracold dipolar bosons in a lattice, we define an order parameter

$$
\Delta=\sum_{k}\left(\frac{\lambda_{k}}{N}\right)^{2},
$$

where $\lambda_{k}$ is the $k^{\text {th }}$ natural occupation [cf. Eq. (2)]. For the superfluid phase, only one eigenvalue $\lambda_{1}$ is non-negligible and, hence, $\Delta=1$. For the Mott insulator as many eigenvalues as there are sites in the lattice are contributing equally, while the rest are negligible and thus $\Delta=\frac{1}{S}$. The crystal state is characterized by $\Delta=\frac{1}{N}$; each of the $N$ bosons occupies a separate orbital, while the other occupations $\lambda_{k}$ with $k>N$ are negligible.

We stress that the Tonks-Girardeau (TG) gas is an intermediate stage to the MI $\rightarrow$ CS transition. While there exists a universal behavior of the natural occupations for the SF, MI, and the CS phases, there is none for the TG gas. Hence, unlike in the case of the SF, MI, and CS states, there is no value of $\Delta$ associated with the TG gas.

We plot the order parameter $\Delta$ as a function of the barrier height $V$ and the interaction strength $g_{d}$ in Fig. 3, thereby constructing a few-body analog of the phase diagram. The superfluid phase is restricted to small values of $V$ and $g_{d}$. The Mott insulator emerges for increasing dipolar interactions. The occurrence of maximal fragmentation marks the emergence of the crystal phase where the short-ranged contribution of the dipolar interactions saturates while the long-ranged contribution [64] of the dipolar interaction potential forces the bosons to become fully separated and fragmented.

A fundamental difference between the transition SF $\rightarrow$ MI and the transition $\mathrm{MI} \rightarrow \mathrm{CS}$ is the mechanism of fragmentation. In the SF $\rightarrow$ MI transition, fragmentation is extrinsic because it is governed by the one-body lattice potential of the Hamiltonian. Here, the eigenvalues of the reduced density matrix are dependent on the number of lattice sites. In the transition MI $\rightarrow$ CS, fragmentation is intrinsic because it is governed exclusively by the dipolar two-body interaction in the Hamiltonian and is not dependent on the one-body potential. Here, the eigenvalues of the reduced density matrix do not depend on the number of lattice sites. The crystal state formation is thus a genuine many-body effect. This explains why the crystallization is seen also in other one-dimensional systems, irrespective of boundary conditions or the one-body potential $[20,24,25,41,43]$.

While we explicitly present the result for a few-body system, we stress that our order parameter is absolutely general and valid for any large but finite system, like the ones produced in cold-atom experiments. Thus, it can, in

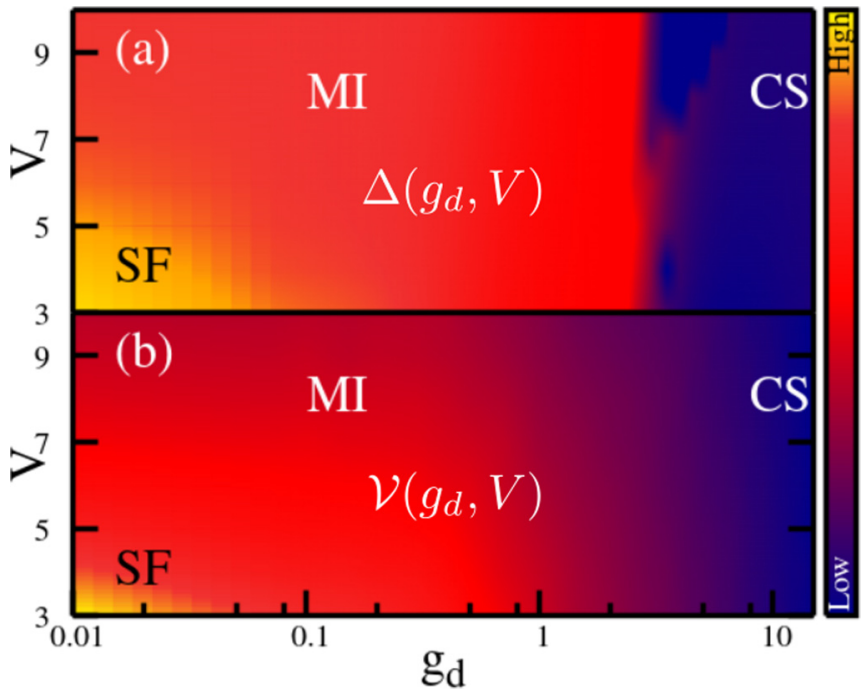

FIG. 3. (a) Phase diagram showing the order parameter $\Delta\left(g_{d}, V\right)$ as a function of the lattice depth $V$ and the dipolar interaction strength $g_{d}$. The values of $\Delta$ for the superfluid (SF), the Mott insulator (MI), and for the crystal state (CS) are, respectively, $\Delta \approx 1$, $\Delta \approx \frac{1}{3}$, and $\Delta \approx \frac{1}{6}$. The SF phase can be observed for a small barrier heights $V$ and low interaction strength $g_{d}$. As $V$ increases, the $\mathrm{SF} \rightarrow \mathrm{MI}$ transition occurs. As $g_{d}$ increases, the MI $\rightarrow$ CS transition takes place. The CS is a maximally fragmented state which forms as a result of dominating long-range interactions. Unlike the $\mathrm{SF} \rightarrow \mathrm{MI}$ transition, the $\mathrm{MI} \rightarrow \mathrm{CS}$ transition is almost independent of the barrier height because the crystal transition is a genuine many-body effect. (b) Simulation of the phase diagram using the variance of single shots in real space $\mathcal{V}\left(g_{d}, V\right)$. For every point, the variance of 10000 single-shot images were computed. The value of the variance $\mathcal{V}$ is maximal for the SF phase, decreases in the MI phase, and attains its minimum value for the CS. The phase diagram using $\mathcal{V}\left(g_{d}, V\right)$ shows all the three phases and closely resembles the one obtained using the order parameter $\Delta\left(g_{d}, V\right)$.

principle, be used to characterize and analyze the phases occurring in such experiments. In the thermodynamic limit $N \rightarrow \infty$ and $S \rightarrow \infty$, we find $\Delta \rightarrow 0$ for both the MI and CS phases. However, $\Delta$ approaches zero with different rates $\tau$, determined by $N / S$ ratio, for the MI and the CS cases. While the order parameter $\Delta$ does coincide for the MI and CS phases in the thermodynamic limit, the finite-size scaling of the rate $\tau$ at which $\Delta$ approaches zero with the system size $\left(\frac{N}{S} \neq 1\right)$ still reveals if the system is in the CS or the MI state.

\section{DETECTION PROTOCOL USING SINGLE SHOTS}

While the order parameter $\Delta$ reflects each quantum phase, the detection of $\Delta$ or the eigenvalues $\lambda_{k}$ of the reduced one-body density matrix remains an experimental challenge. Further, the direct measurement of the densities of the system with a resolution sufficient to detect intrasite features also represents a formidable problem; this problem can be solved since the lattice constant is experimentally tunable and intrawell structures are thus resolvable [71].

Experimental absorption images measure the positions of all particles simultaneously. A single absorption image or 
a single-shot measurement contains information about the correlations of the atoms in Bose-Einstein condensates [72]. In particular, the variance of single-shot measurements in momentum space has been found to yield valuable information on the natural occupations [73].

To simulate such an absorption image, we use the wave functions $\Psi\left(x_{1}, \ldots, x_{N}\right)$ obtained from our MCTDHB simulations as a starting point. A single-shot measurement corresponds to drawing all particle positions $\left(s_{1}, \ldots, s_{N}\right)$ simultaneously from the probability $\left|\Psi\left(x_{1}, \ldots, x_{N}\right)\right|^{2}$. We use the algorithm documented in Refs. [72,73] to draw $N_{\text {shots }}$ samples from a given state $\Psi\left(x_{1}, \ldots, x_{N}\right)$. For every simulated singleshot measurement, i.e., every single sample which is drawn, a convolution with a three-pixels-wide Gaussian is performed to emulate the point spread function of a realistic imaging system. The result of these convolutions are $N_{\text {shots }}$ functions $\left\{\mathcal{B}_{j}(x)\right\}_{j=1}^{N_{\text {shots }}}$. From these functions $\mathcal{B}_{j}(x)$ obtained from $N_{\text {shots }}$ single shots, the single-shot variance $\mathcal{V}$ is obtained as follows:

$$
\begin{aligned}
\mathcal{V} & =\int d x \frac{1}{N_{\text {shots }}} \sum_{j=1}^{N_{\text {shots }}}\left[\mathcal{B}_{j}(x)-\overline{\mathcal{B}}(x)\right]^{2}, \\
\overline{\mathcal{B}}(x) & =\frac{1}{N_{\text {shots }}} \sum_{j=1}^{N_{\text {shots }}} \mathcal{B}_{j}(x) .
\end{aligned}
$$

$\mathcal{V}$ represents the average variance of each single-shot measurement from its mean value integrated in space. In the following, we discuss how to detect the emerging phases [Fig. 3(a)] using single-shot measurements. Here, we adopt a similar approach to the one taken in Ref. [73] and compute the variance of simulations of single-shot measurements in real space. We use spatial instead of momentum measurements because the spatial single-shot distributions let us access the degree of localization of the bosons. In the present system of dipolar bosons in a lattice, the degree of localization increases with increasing dipolar interaction strength [Fig. 3(b)]: in the superfluid, the atoms are delocalized in the entire lattice. The single-shot variance $\mathcal{V}$ is largest for this state since the atoms can be sampled from any position in the lattice. In the Mott insulator, the atoms localize in individual lattice sites while they are delocalized within each lattice site. As a consequence, the variance in the single-shot measurements decreases as compared to the superfluid. In the crystal phase, the atoms localize in individual natural orbitals, i.e., they form a structure irrespective of the underlying lattice potential. This further localization through maximizing the fragmentation further minimizes the variance in single-shot measurements. In summary, we find that the single-shot variance $\mathcal{V}$ is able to detect all the few-body phases observed and the phase diagram obtained using $\mathcal{V}\left(g_{d}, V\right)$ [Fig. 3(b)] shows qualitative similarity with that obtained with $\Delta\left(g_{d}, V\right)$ [Fig. 3(a)]. Most importantly, the crystal state is clearly discernible using the single-shot variance $\mathcal{V}$. Since our simulations of single-shot measurements correspond to standard experimental absorption imaging, we thus have shown a straightforward technologically achievable way to detect the emergent phases. Note that since we investigate a small system, the phase transitions are not expected to be sharp. The phase transitions of the system are smoothened in single-shot measurements as compared to the order parameter $\Delta$ because in the transitions between the phases in a finite system, the orbitals which are delocalized between sites become occupied. Moreover, the large fluctuations present in our small finite-size system lead to deviations between the phase boundaries obtained from the order parameter $\Delta$ and the single-shot variance $\mathcal{V}$.

\section{CONCLUSIONS}

In this work, we have explored the emergent phases of a strongly interacting finite ensemble of dipolar bosons in an optical lattice. As the interaction increases from weak to strong, the dipolar bosons undergo a transition from a superfluid to a Mott insulator and eventually to the crystal state. We analyzed the one- and two-body densities that reveal the characteristic signatures of these transitions. We then established a general order parameter that characterizes all the emergent phases in a finite ensemble of ultracold few dipolar bosons in commensurately filled lattices in one spatial dimension. Using this order parameter, we constructed a phase diagram for the few-body system as a function of interaction strength and lattice depth and discussed its fundamental connection to the one- and two-body densities of the system. Finally, we demonstrated an experimental protocol using the single-shot variance that provides a procedure to detect all the emergent few-body phases of the system using standard absorption imaging. Since both our order parameter as well as our detection protocol are not fundamentally restricted to very small systems, they may be expected to be valid for larger systems like typical cold-atom experiments as hinted by our results in Appendix A. Our work provides a detailed understanding of the strong interaction regimes of one-dimensional dipolar bosons in lattices with commensurate filling. Investigations into incommensurate fillings and two-dimensional lattice systems are possible extensions of this work.

\section{ACKNOWLEDGMENTS}

B.C. gratefully acknowledges the financial support from Department of Science and Technology, Government of India, under DST Inspire Faculty fellowship. A.U.J.L. acknowledges financial support by the Swiss SNF and the NCCR Quantum Science and Technology, the Austrian Science Foundation (FWF), under Grant No. F65 (SFB "Complexity in PDEs"), and the Wiener Wissenschafts- und TechnologieFonds (WWTF) Project No. MA16-066 ("SEQUEX"). Computation time on the Hazel Hen cluster of the HLRS in Stuttgart and the HPC2013 cluster of the IIT Kanpur are gratefully acknowledged. B.C. thanks A. K. Agarwal, IITK, for help with an initial computational resource.

\section{APPENDIX A: RESULTS FOR DIFFERENT SYSTEM PARAMETERS}

In this Appendix, we show the Fig. 1 results of the main text for larger lattice size $S=5$ as well as larger filling of three bosons per site to demonstrate that the results obtained in the main text can be generalized. In a comparison of the results in the main text to results without the short-ranged 

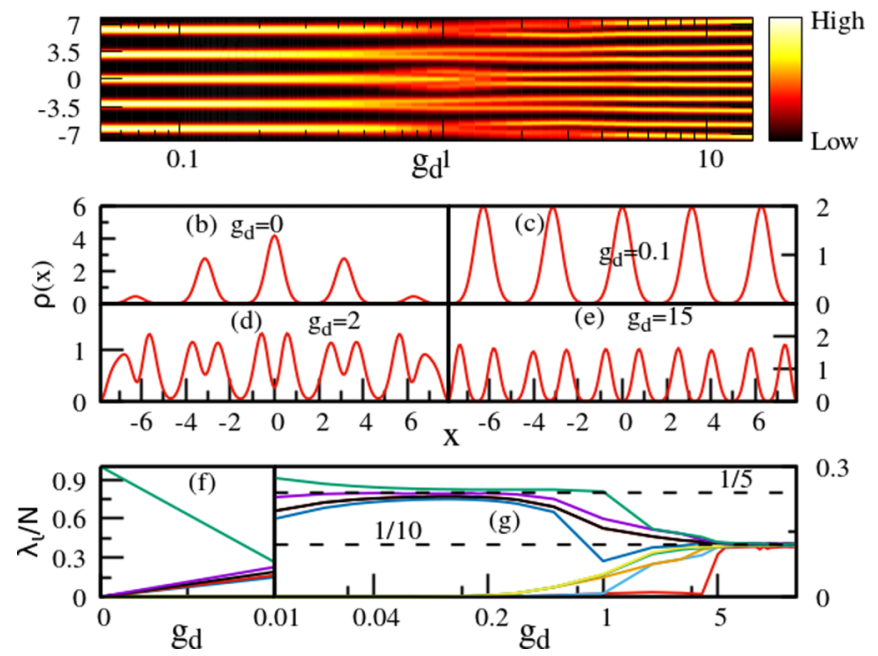

FIG. 4. Results of Fig. 1 for larger lattice size $(S=5)$. Top panel: one-body density $\rho(x)$ for $S=5, N=10$ plotted as a function of space $x$ and dipolar coupling $g_{d}$ for $V=8$. For small $g_{d}$ the density exhibits a fivefold splitting corresponding to the $S=5$ lattice sites as we are in MI state. As the dipolar interaction strength $g_{d}$ increases, a transition from a fivefold spatial splitting to a tenfold spatial splitting corresponding to $N=10$ bosons takes place as we transition to the crystal state. (b)-(e) One-body densities representative for different phases. (b) $g_{d}=0$, the superfluid phase with a maximal population at the center. (c) $g_{d}=0.1$, the Mott-insulating phase. Five peaks correspond to localization in each well with each site having two particles. (d) $g_{d}=2.0$, fermionization and mimicking of the Tonks gas can be seen from the characteristic dips in the density within each well. (e) $g_{d}=15.0$, the crystal state formation can be seen from the emergence of well-separated density peaks as the long-ranged interaction starts to dominate. (f), (g) Normalized population of natural orbitals as a function of the interaction strength $g_{d}$. The superfluid $\left(g_{d} \approx 0\right)$ is condensed and only one orbital contributes significantly. For the Mott insulator as many orbitals as there are sites $(S=5)$ contribute, all having an equal population of $\approx 0.2$. For large $g_{d}$ as many orbitals as there are particles $(N=10)$ contribute with an equal population of $\approx 0.1$ and the system reaches the maximally fragmented crystal state.

contribution, $g_{c} \delta\left(x-x^{\prime}\right)$, we confirmed that no qualitative differences are triggered by the inclusion of the additional short-ranged contribution. We therefore neglect the additional short-ranged contribution $g_{c} \delta\left(x-x^{\prime}\right)$ in this Appendix.

\section{Different lattice sizes}

To demonstrate the generality of our results for three wells and six particles in the main text also for larger lattices, we show results for five lattice sites $(S=5)$ and ten particles $(N=10)$ in Fig. 4. Similar to the main-text results, the onebody density evolves from an initial superfluid phase to a Mott-insulator phase, characterized by five separate density peaks (corresponding to $S=5$ lattice sites). Stronger interactions result in intrawell splitting of the bosons as a result of the repulsive dipolar interaction. Finally, at the crystal phase, ten separated density peaks are seen corresponding to $N=10$ particles.
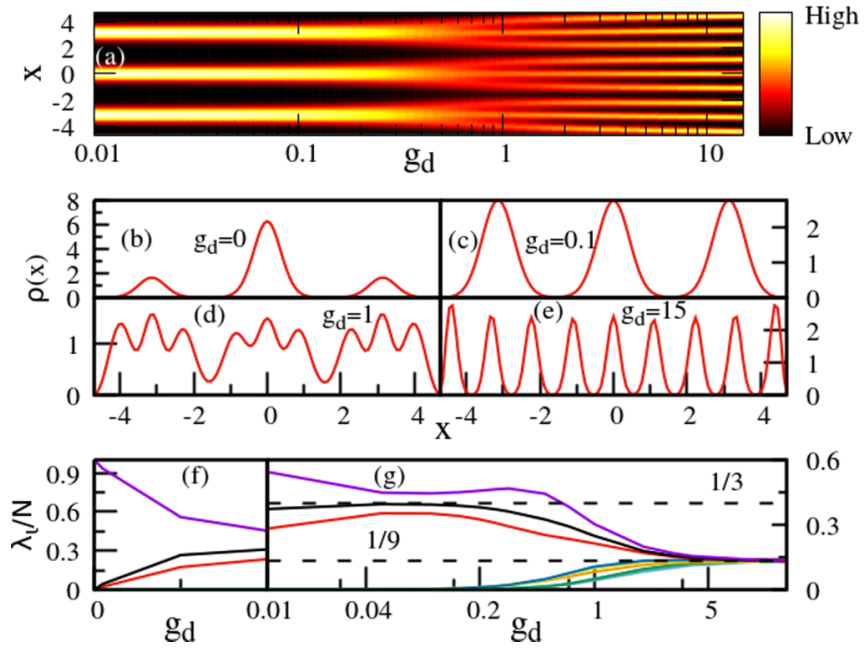

FIG. 5. Results of Fig. 1 for higher filling factor $(S=3, N=9)$. Top panel: one-body density $\rho(x)$ for $S=3, N=9$ plotted as a function of space $x$ and dipolar coupling $g_{d}$ for $V=8$. For small $g_{d}$ in the superfluid and Mott-insulating phases, the density is split threefold because the lattice has three sites $(S=3)$. Since the filling factor is $v=3$ with a total of $N=9$ bosons, the density splits into three parts at each well, forming an overall ninefold split structure in the crystal phase, as interactions become stronger. (b)-(e) Onebody densities representative for different phases. (b) $g_{d}=0$, the superfluid with a maximal population in the central well. (c) $g_{d}=$ 0.1 , the Mott insulator shows localization in each site forming three density peaks. (d) $g_{d}=1.0$, the mimicking of the Tonks gas is seen from the emergence of characteristic dips that result in a three-hump structure within every site. (e) $g_{d}=15.0$, the crystal state formation can be seen from the emergence of well-separated density peaks for each particle as the long-ranged interaction starts to dominate. (f), (g) Normalized population of natural orbitals as a function of the interaction strength $g_{d}$. In the superfluid phase $\left(g_{d} \approx 0\right)$, the system is condensed and only one orbital contributes significantly. For the Mott insulator as many orbitals as there are sites $(S=3)$ contribute, all having approximately equal populations of $\approx 0.33$. For large $g_{d}$, in the crystal phase, as many orbitals as there are particles $(N=9)$ are populated equally having population $\approx 0.11$.

It is thus seen that the conjectures of the main text are valid also for larger lattices with commensurate filling.

\section{Different filling factors}

To demonstrate the generality of our results in the main text for two particles per lattice site also for different commensurate fillings, we show results for a triple-well lattice $(S=3)$ with nine particles $(N=9)$, i.e., a filling of three particles per site in Fig. 5.

Here, the transition from the SF to MI phase is exhibited with three density peaks corresponding to $S=3$ lattice sites. With increasing interaction, since each lattice site is triply occupied, we see a threefold splitting in each well. At the crystal phase, the splitting is complete and we obtain nine density peaks corresponding to $N=9$. It is thus seen that the findings documented in the main text are true also for different commensurate filling factors. 


\section{APPENDIX B: MOMENTUM DISTRIBUTION}

In this Appendix we analyze the one-particle momentum density

$$
\tilde{\rho}(k)=2 \pi\left\langle k\left|\rho_{1}\right| k\right\rangle=\int d x \int d x^{\prime} e^{-i k\left(x-x^{\prime}\right)} \rho^{1}\left(x, x^{\prime}\right) .
$$

Figure 6 shows the momentum density $\tilde{\rho}$ as a function of $k$ and $g_{d}$ for $V=8$. For very weak interactions $g_{d} \approx 0$, the momentum density has a sharp central peak at $k=0$, with additional smaller peaks on the side. This is due to the fact that since the bosons are spatially delocalized over the lattice: the momentum distribution (which is the Fourier transform of density), consequently, are localized and show Bragg peaks near the reciprocal lattice vectors $k=2 \pi / a(a$ being the lattice spacing). As the strength of the interactions increases, the increasing spatial localization of the bosons in the MI phase manifests in the smearing of the central peak and suppression of the Bragg peaks. This demonstrates the reduction of coherence in the MI phase. Further increasing the strength of interactions triggers no qualitative change in $\tilde{\rho}$. The only quantitative change is a spreading of $\tilde{\rho}$, indicating further spatial localization and corresponding momentum delocalization as the system reaches the crystal state. We note here the absence of a clear signature of the phase transition $\mathrm{MI} \rightarrow \mathrm{CS}$ in the momentum distributions $\tilde{\rho}$. Hence, the momentum distribution is not a good candidate to observe the phase transition to the crystal phase.
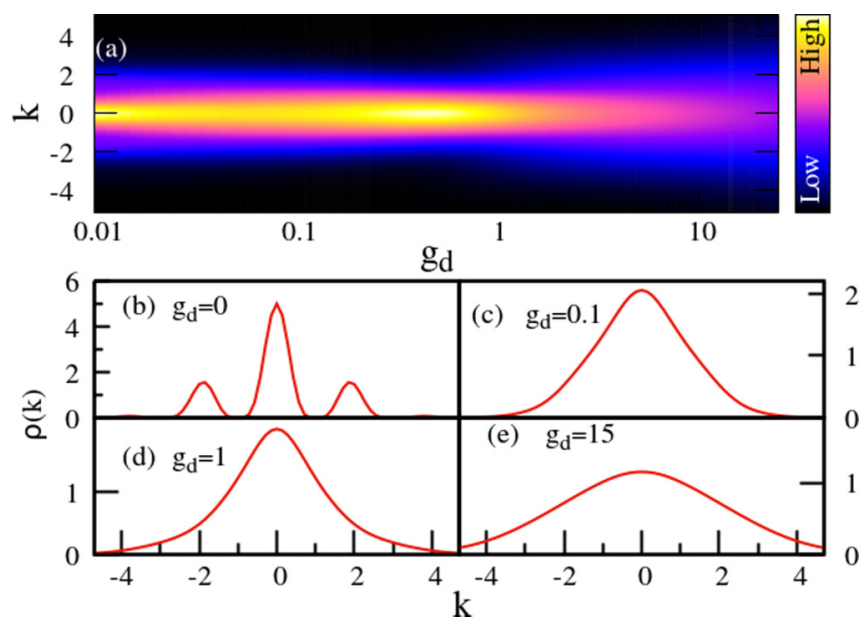

FIG. 6. Tracing the evolution of the momentum density as a function of dipole-dipole interaction strength. (a) Momentum density profile $\tilde{\rho}(k)$ as a function of interaction strength $g_{d}$. The initially centrally peaked momentum density shows spreading as the strength of the dipole-dipole interactions increase. (b)-(e) Shows $\tilde{\rho}(k)$ at different interaction strengths characteristic to the emergent phases [cf. Figs. 1(b)-1(e) in the main text]. At $g_{d}=0$ there is a central sharp peak, with smaller peaks near the reciprocal lattice vectors. With increasing $g_{d}$, the small side peaks are suppressed while the central peak becomes flattened. The crystal state at $g_{d}=15$ features a central peak that is strongly spread to a plateaulike structure.
[1] M. A. Baranov, Phys. Rep. 464, 71 (2008).

[2] T. Lahaye, C. Menotti, L. Santos, M. Lewenstein, and T. Pfau, Rep. Prog. Phys. 72, 126401 (2009).

[3] A. Griesmaier, J. Werner, S. Hensler, J. Stuhler, and T. Pfau, Phys. Rev. Lett. 94, 160401 (2005).

[4] Q. Beaufils, R. Chicireanu, T. Zanon, B. Laburthe-Tolra, E. Maréchal, L. Vernac, J.-C. Keller, and O. Gorceix, Phys. Rev. A 77, 061601 (2008).

[5] M. Lu, N. Q. Burdick, S. H. Youn, and B. L. Lev, Phys. Rev. Lett. 107, 190401 (2011).

[6] K. Aikawa, A. Frisch, M. Mark, S. Baier, A. Rietzler, R. Grimm, and F. Ferlaino, Phys. Rev. Lett. 108, 210401 (2012).

[7] K. K. Ni, S. Ospelkaus, M. H. G. de Miranda, A. Pe'er, B. Neyenhuis, J. J. Zirbel, S. Kotochigova, P. S. Julienne, D. S. Jin, and J. Ye, Science 322, 231 (2008).

[8] J. W. Park, S. A. Will, and M. W. Zwierlein, Phys. Rev. Lett. 114, 205302 (2015).

[9] S. Yi and L. You, Phys. Rev. A 63, 053607 (2001).

[10] L. Santos, G. V. Shlyapnikov, P. Zoller, and M. Lewenstein, Phys. Rev. Lett. 85, 1791 (2000).

[11] K. Góral, K. Rzążewski, and T. Pfau, Phys. Rev. A 61, 051601 (2000).

[12] C. Eberlein, S. Giovanazzi, and D. H. J. O’Dell, Phys. Rev. A 71, 033618 (2005).

[13] K. Góral and L. Santos, Phys. Rev. A 66, 023613 (2002).

[14] T. Koch, T. Lahaye, J. Metz, B. Fröhlich, A. Griesmaier, and T. Pfau, Nat. Phys. 4, 218 (2008).
[15] K. Góral, L. Santos, and M. Lewenstein, Phys. Rev. Lett. 88 170406 (2002).

[16] C. Menotti, C. Trefzger, and M. Lewenstein, Phys. Rev. Lett. 98, 235301 (2007).

[17] B. Capogrosso-Sansone, C. Trefzger, M. Lewenstein, P. Zoller, and G. Pupillo, Phys. Rev. Lett. 104, 125301 (2010).

[18] G. M. Bruun and E. Taylor, Phys. Rev. Lett. 101, 245301 (2008).

[19] N. R. Cooper and G. V. Shlyapnikov, Phys. Rev. Lett. 103, 155302 (2009).

[20] A. S. Arkhipov, G. E. Astrakharchik, A. V. Belikov, and Y. E. Lozovik, JETP Lett. 82, 39 (2005).

[21] R. Citro, E. Orignac, S. De Palo, and M. L. Chiofalo, Phys. Rev. A 75, 051602(R) (2007).

[22] S. De Palo, E. Orignac, R. Citro, and M. L Chiofalo, Phys. Rev. B 77, 212101 (2008).

[23] P. Pedri, S. De Palo, E. Orignac, R. Citro, and M. L. Chiofalo, Phys. Rev. A 77, 015601 (2008).

[24] S. Zöllner, G. M. Bruun, C. J. Pethick, and S. M. Reimann, Phys. Rev. Lett. 107, 035301 (2011).

[25] S. Zöllner, Phys. Rev. A 84, 063619 (2011).

[26] M. Maik, P. Buonsante, A. Vezzani, and J. Zakrzewski, Phys. Rev. A 84, 053615 (2011).

[27] F. Serwane, G. Zürn, T. Lompe, T. B. Ottenstein, A. N. Wenz, and S. Jochim, Science 332, 336 (2011).

[28] S. Murmann, A. Bergschneider, V. M. Klinkhamer, G. Zürn, T. Lompe, and S. Jochim, Phys. Rev. Lett. 114, 080402 (2015). 
[29] S. Murmann, F. Deuretzbacher, G. Zürn, J. Bjerlin, S. M. Reimann, L. Santos, T. Lompe, and S. Jochim, Phys. Rev. Lett. 115, 215301 (2015)

[30] A. U. J. Lode, K. Sakmann, O. E. Alon, L. S. Cederbaum, and A. I. Streltsov, Phys. Rev. A 86, 063606 (2012).

[31] E. Fasshauer and A. U. J. Lode, Phys. Rev. A 93, 033635 (2016).

[32] I. Brouzos, S. Zöllner, and P. Schmelcher, Phys. Rev. A 81, 053613 (2010).

[33] O. E. Alon, A. I. Streltsov, and L. S. Cederbaum, Phys. Rev. Lett. 95, 030405 (2005).

[34] R. Roy, A. Gammal, M. C. Tsatsos, B. Chatterjee, B. Chakrabarti, and A. U. J. Lode, Phys. Rev. A 97, 043625 (2018).

[35] R. Landig, L. Hruby, N. Dogra, M. Landini, R. Mottl, T. Donner, and T. Esslinger, Nature (London) 532, 476 (2016).

[36] G. Jotzu, M. Messer, R. Desbuquois, M. Lebrat, T. Uehlinger, D. Greif, and T. Esslinger, Nature (London) 515, 237 (2014).

[37] N. Goldman, J. C. Budich, and P. Zoller, Nat. Phys. 12, 639 (2016).

[38] C. Gross and I. Bloch, Science 357, 995 (2017).

[39] E. G. Dalla Torre, E. Berg, and E. Altman, Phys. Rev. Lett. 97, 260401 (2006)

[40] X. Deng and L. Santos, Phys. Rev. B 84, 085138 (2011).

[41] G. E. Astrakharchik, G. E. Morigi, G. De Chiara, and J. Boronat, Phys. Rev. A 78, 063622 (2008).

[42] G. E. Astrakharchik and Yu. E. Lozovik, Phys. Rev. A 77, 013404 (2008).

[43] F. Deuretzbacher, J. C. Cremon, and S. M. Reimann, Phys. Rev. A 81, 063616 (2010).

[44] B. Chatterjee, I. Brouzos, L. Cao, and P. Schmelcher, J. Phys. B: At. Mol. Opt. Phys. 46, 085304 (2013).

[45] C. Yannouleas and U. Landman, Rep. Prog. Phys. 70, 2067 (2007).

[46] D. Peter, K. Pawlowski, T. Pfau, and K. K. Rzążewski, J. Phys. B: At. Mol. Opt. Phys. 45, 225302 (2012).

[47] T. Lahaye, T. Pfau, and L. Santos, Phys. Rev. Lett. 104, 170404 (2010).

[48] L. Dell'Anna, G. Mazzarella, V. Penna, and L. Salasnich, Phys. Rev. A 87, 053620 (2013).

[49] B. Xiong and U. R. Fischer, Phys. Rev. A 88, 063608 (2013).

[50] A. Gallemi, M. Guilleumas, R. Mayol, and A. Sanpera, Phys. Rev. A 88, 063645 (2013).

[51] A. Gallemi, G. Queralto, M. Guilleumas, R. Mayol, and A. Sanpera, Phys. Rev. A 94, 063626 (2016).

[52] L. Cao, S. I. Mistakidis, X. Deng, and P. Schmelcher, Chem. Phys. 482, 303 (2017).
[53] Q.-S. Tan, H.-Y. Lu, and S. Yi, Phys. Rev. A 93, 013606 (2016).

[54] A. I. Streltsov, Phys. Rev. A 88, 041602(R) (2013).

[55] H. Y. Lu and S. Yi, Sci. China Phys. Mech. Astron. 55, 1535 (2012).

[56] O. E. Alon, A. I. Streltsov, and L. S. Cederbaum, Phys. Rev. A 77, 033613 (2008).

[57] A. U. J. Lode, M. C. Tsatsos, E. Fasshauer, R. Lin, L. Papariello, P. Molignini, and C. Lévêque, MCTDH-X: The timedependent multiconfigurational Hartree for indistinguishable particles software, http://ultracold.org (2018).

[58] A. U. J. Lode, Phys. Rev. A 93, 063601 (2016).

[59] S. Sinha and L. Santos, Phys. Rev. Lett. 99, 140406 (2007).

[60] Y. Cai, M. Rosenkranz, Z. Lei, and W. Bao, Phys. Rev. A 82, 043623 (2010).

[61] M. Greiner, O. Mandel, T. Esslinger, T. W. Hänsch, and I. Bloch, Nature (London) 415, 39 (2002).

[62] D. Jaksch, C. Bruder, J. I. Cirac, C. W. Gardiner, and P. Zoller, Phys. Rev. Lett. 81, 3108 (1998).

[63] U. R. Fischer, A. U. J. Lode, and B. Chatterjee, Phys. Rev. A 91, 063621 (2015).

[64] For a lattice potential, the "short-range part of the dipolar interaction" refers to the part of the interaction potential that is confined within a single site; it can be quantified using an integral of the interaction potential over one site $V_{\mathrm{S}} \equiv \int_{0}^{\frac{\pi}{2}} \frac{g_{d}}{x^{3}+\alpha} d x$. The "long-range part of the interaction" refers to the part of the interaction potential in the domain excluding the local site; it can be quantified using an integral of the interaction potential on all but the local site $V_{\mathrm{L}} \equiv \int_{\frac{\pi}{2}}^{\infty} \frac{g_{d}}{x^{3}+\alpha} d x$.

[65] D. S. Lühmann, K. Bongs, K. Sengstock, and D. Pfannkuche, Phys. Rev. A 77, 023620 (2008).

[66] M. Girardeau, J. Math. Phys. 1, 516 (1960).

[67] The exact mapping is valid for $\alpha=0$ exploiting the divergence at $x_{i}=x_{j}$. Since in our case $\alpha \neq 0$, the mapping is not valid in a strict sense. For sufficiently large $g_{d}$, the fermionization effect can, however, be seen clearly in our results.

[68] O. Penrose and L. Onsager, Phys. Rev. 104, 576 (1956).

[69] R. W. Spekkens and J. E. Sipe, Phys. Rev. A 59, 3868 (1999).

[70] E. J. Mueller, T.-L. Ho, M. Ueda, and G. Baym, Phys. Rev. A 74, 033612 (2006).

[71] R. Gati, M. Albiez, J. Fölling, B. Hemmerling, and M. K. Oberthaler, Appl. Phys. B 82, 207 (2006).

[72] K. Sakmann and M. Kasevich, Nat. Phys. 12, 451 (2016).

[73] A. U. J. Lode and C. Bruder, Phys. Rev. Lett. 118, 013603 (2017). 